\title{
Gastrointestinal complications in patients with diabetes mellitus
}

\author{
Agnieszka E. Zawada ${ }^{A, B, D}$, Małgorzata Moszak ${ }^{B}$, Dorota Skrzypczak ${ }^{B}$, Marian Grzymisławski ${ }^{A, E, F}$ \\ Department and Clinic of Internal and Metabolic Diseases and Dietetics, Poznan University of Medical Sciences, Poland \\ A - research concept and design; B - collection and/or assembly of data; C - data analysis and interpretation; \\ $D$ - writing the article; $E$ - critical revision of the article; $F$ - final approval of the article
}

Address for correspondence

Agnieszka E. Zawada

E-mail:aga.zawada@gmail.com

Funding sources

None declared

Conflict of interest

None declared

Received on August 16, 2016

Reviewed on September 7, 2016

Accepted on December 20, 2016

\begin{abstract}
Diabetes mellitus is a systemic disease which affects patients of various age. Hyperglycemia induces damage of vascular endothelium, development of chronic inflammation, organic and functional lesions in several systems and organs. The principal gastroenterological complaints linked to the manifestation of the disease include abdominal pain, diarrhea, nausea, flatulence, and vomiting. However, complications in the alimentary system may manifest exclusively by difficulties in reaching normoglycemia and numerous persistent episodes of hypoglycemia. The most frequent complication of diabetes mellitus affecting the alimentary tract involves gastroparesis and disturbances in pancreatic function. Diabetes may also aggravate other coexisting diseases, such as gastroesophageal reflux or periodontitis. Subject-based references accentuate also a significantly more frequent manifestation together with diabetes of other autoimmune diseases, such as celiac disease or autoimmune gastritis. Also, a hepatic microangiopathy and increased incidence of certain tumors, linked to the manifestation of insulin resistance, may be regarded to represent complications of long-term diabetes. Rapid diagnosis and adequate treatment may significantly improve a patient's quality of life and influence the prolonged control of glycemia. Nevertheless, this requires a rigorous analysis of the signs and clinical condition of a patient as well as individualization of recommendations and therapy.
\end{abstract}

Key words: diabetes mellitus, gastrointestinal disorders, insulin resistance

DOI

10.17219/acem/67961

\section{Copyright}

Copyright by Author(s)

This is an article distributed under the terms of the

Creative Commons Attribution Non-Commercial License

(http://creativecommons.org/licenses/by-nc-nd/4.0/) 
Diabetes mellitus (DM) represents a disease affecting an increasingly large group of people. Around 415 million people worldwide are estimated to be affected and by 2040 the number of such patients is thought to reach around 642 million. ${ }^{1}$ The principal hazard for the patient linked to the disease involves chronic complications of micro- and macropathy character: they induce severe organic dysfunctions and may lead to a sudden death of the patient. Troublesome, even if usually not linked to direct hazard of death, are alimentary disturbances. They used to develop in patients with long-lasting diabetes mellitus, frequently uncontrolled and metabolically unbalanced for many years. The abnormalities may affect practically any fragment of alimentary system. Signs/symptoms such as nausea, vomits, disturbed swallowing, abdominal pain, disturbed absorption, diarrhea, obstipation may represent prodromes of another, coexisting disease or they may develop in the course of metabolically uncontrolled diabetes mellitus.

The link with diabetes mellitus manifests also more frequently in developing infections, functional and organic disturbances and some tumors. Moreover, poorly controlled diabetes may result in lesions in central and peripheral nerve fibers, which renders relevant signs/symptoms variable and their diagnosis, therefore, poses a challenge for physicians-practitioners.

\section{Diseases in oral cavity as an index of metabolically unbalanced diabetes mellitus}

Diabetes mellitus results in the appearance of problems already in the preliminary fragment of alimentary tract. The main diseases in the oral cavity include fungal infections and periodontal pathology. Prodromes of such diseases include inconveniences, such as dry oral cavity, reddening and hyperemia of throat, and atrophic lesions on the tongue. The most frequent infections of the oral cavity include candidiasis, accounting for 40-60\% infections of the oral cavity. Studies conducted by Shenoy et al. demonstrated significantly more frequent infections with Candida in groups of patients with diabetes type 1 (DM1) - 30\% or diabetes type 2 (DM2) - 33\% infections, as compared to diabetes-free patients $-7 \%$ infections. $^{2}$ The values of CFU/mL were also significantly higher in the groups of DM1 and DM2 than among healthy individuals. The study demonstrated also a significant positive relationship between $\mathrm{CFU} / \mathrm{mL}$ on one hand and fasting glycemia and $\mathrm{HbA}_{1 \mathrm{c}}$ level, on the other. ${ }^{2}$ The course and intensity of candidiasis reflect also the intensity of disturbances in carbohydrate metabolism. In patients with a pre-diabetic condition, candidiasis manifested a statistically more severe course (evaluated on the basis of standard laboratory techniques and by dental defects) than in healthy patients. ${ }^{3}$ Another disease of the oral cavity which used to affect diabetic patients involves periodontitis. It develops 2.6 times more frequently in diabetic patients than in the healthy population, and in persons with a metabolically unbalanced diabetes this incidence continues to increase (up to 2.9 -fold higher one). ${ }^{4,5}$ In parallel, it can be concluded that the relationship is bilateral: diabetes aggravates periodontitis and makes it more difficult to cure, while the chronic inflammatory process intensifies disturbances in carbohydrate metabolism. ${ }^{6}$ In the metaanalysis by Wang et al., conducted on the basis of studies including around 1,135 persons, it was confirmed that after 3 months of intense treatment targeting periodontal diseases, reduced values of $\mathrm{HbA}_{1 \mathrm{c}}$ were detected. ${ }^{7}$ Similar results were obtained by Białecka et al., who recorded a significant reduction in $\mathrm{HbA}_{1 \mathrm{c}} 1$ year following tonsillectomy. ${ }^{8}$ The studies confirmed the need for systematic dental control and intense treatment of any inflammatory foci in the oral cavity in order to gain metabolic control of diabetes mellitus.

\section{Diabetic disturbances in function of esophagus}

One of the principal disturbances related to the esophagus in diabetic patients involves manifestation of a peristaltic wave (low amplitude of contractions and contractions of third order) with a delayed passage and a dysfunction of the lower sphincter (a decreased tension of LES). There exists data indicating that the complication develops in as many as $75 \%$ diabetic patients with a coexisting autonomic neuropathy. Principal symptoms manifested in patients with DM include dysphagia and esophageal burning. Obesity and insulin resistance which accompany DM2 represent recognized risk factors of Barrett's esophagus development, of progression of already manifested inflammatory lesions and intensification of dysphagia. ${ }^{9}$ The relationship between obesity, diabetes and insulin resistance on one hand and an increased incidence of esophageal adenocarcinomas was confirmed in several reports. ${ }^{10,11}$ In studies conducted on 2,836 veterans, diabetic patients were found to suffer more frequently from esophageal and cardiac adenocarcinomas. In a model of logistic regression, the relationship was documented between incidence of esophageal adenocarcinoma (EAC) on one hand and age, black race and tobacco smoking on the other. However, the increase in incidence of EAC in diabetic patients proved to be independent of coexisting obesity in this group of patients. The study confirmed also more frequent manifestation of EAC in persons with gastroesophageal reflux disease (GERD) at high values of glycosylated hemoglobin. ${ }^{12}$ It should be mentioned also that other frequently developing complications of diabetes, such as gastroparesis, also promotes the development of gastro-esophageal reflux, which also represents an independent risk factor of adenocarcinoma development. ${ }^{13}$ 


\section{Gastric disturbances in the course of diabetes mellitus}

One of the more frequently developing complications of diabetes involves gastroparesis. It involves a syndrome of signs/symptoms related to upper fragment of alimentary tract reflecting disturbances in stomach emptying. Epidemiological data indicates that the complication (used to reflect disturbances of autonomic system) develops in $5-12 \%$ patients with diabetes and significantly more frequently in patients already affected by other complications. In the study by Bharucha et al., $47 \%$ of the patients, already affected by other complications, demonstrated delayed emptying of the stomach. ${ }^{14}$ The first and most characteristic symptom of gastroparesis involves nausea, developing in $90 \%$ of patients. Other symptoms, such as early post-alimentary satiety, vomiting, flatulence and the sensation of gastric distension, may induce disturbances in digestion and absorption of food components and difficulties in gaining metabolic control in diabetes. Frequently, no relationship can be detected between the intensity of symptoms and the function of gastric emptying. Nevertheless, in the study by Gourcerol et al., the increasing resistance of pylorus in diabetic patients was found to correlate with the intensity of symptoms and quality of life, evaluated using the gastrointestinal quality of life (GIQLI) questionnaire. ${ }^{15}$ The intensity of gastroparesis manifests a correlation with absence of metabolic balance of diabetes in patients with diabetes type 1 , although it has not been finally proven in patients with type 2 diabetes. ${ }^{16}$ Apart from the neuropathy of vagus nerve, disturbed emptying of the stomach may be induced by acute hyper-and hypoglycemia, hypo- or hyperinsulinemia, disturbed secretion of hormones in alimentary tract, and the more frequent in this group colonization with H. pylori. ${ }^{17,18}$ Histopathological investigations confirmed that patients with gastroparesis, due to persisting chronic inflammatory process, manifest a decreased number of interstitial Cajal cells (ICCs), the number of NO-secreting neurons is reduced, atrophy of smooth muscles is accompanied by presence of lymphocyte infiltrates. ${ }^{19}$ On the other hand, the studies by Choi et al. confirmed presence of macrophages CD206 ${ }^{+}$, which protected against gastroparesis. ${ }^{20}$ The number of such macrophages manifested correlation with number of ICCs.

Apart from a disturbed emptying of stomach, diabetic patients may also manifest a modified contractility of duodenum. In studies by Barshop et al., the duodenal contractile activity was found to correlate more closely with signs/symptoms of gastroparesis than the motoric activity of cardia. ${ }^{21}$ Diagnosis of gastroparesis used to be equivocal, as the reference approach involves isotopic scintigraphy and a patient exhausts the criteria when $2 \mathrm{~h}$ after the start of the test his/her stomach continues to contain at least $60 \%$ of original radioactivity and after $4 \mathrm{~h}$ it contains over $10 \%$ of original radioactivity. External electrogastrography represents a noninvasive and useful technique, but due to the costs involved, it is poorly accessible. The first-line drug in the treatment of the complication include pro-kinetic drugs, such as metoclopramide, itopride and the ghrelin receptor agonist, relamorelin. However, their efficacy is restricted or they are not generally accessible. In the treatment of gastroparesis, the role of frequent consumption of small meals, of a liquid diet, containing low amount of lipids, used to be stressed, as it significantly alleviates symptoms. In addition, proton pump inhibitors and antidepressants such as amitriptyline and escitalopram can be used. In experimental studies attempts were made to use irbesartan, as a drug which reduces leptin concentration, the hormone which significantly delays the process of gastric emptying. However, the obtained results remain equivocal and require further observations. ${ }^{22}$

\section{Small intestine: Celiac disease and changes in intestinal microflora as diseases coexisting with diabetes mellitus}

With increasing frequency, the coexistence of disturbances in absorption is seen to affect patients with type 1 diabetes. Depending on source data, celiac disease coexists in 6 to $15 \%$ of patients with diabetes. In most of the patients, it is diagnosed within 5 years after the diagnosis of diabetes (in 79\% patients, celiac disease is diagnosed within the first 5 years, in 55\% within 2 years and in $40 \%$ within a year after developing diabetes). ${ }^{23}$ However, it is significant that just $10 \%$ of patients present typical clinical signs/symptoms of the disease. The only sign of the disease may involve relapsing hypoglycemias and a poor metabolic balancing of diabetes. Coeliac disease developing in persons with diabetes manifests also a quite distinct course than that developing in diabetes-free individuals. In diabetes, the incidence of celiac disease in men and women is almost the same, without the 3 -fold prevalence of incidence among women, detected in the general population. In order to exclude celiac disease, detection of anti-TTG antibodies is employed as well as genetic studies, allowing for the identification of HLA-DQ2 antigen, present in 90\% of patients with celiac disease and in $55 \%$ of patients with diabetes type 1 . Apart from celiac disease, diabetic patients are also more frequently troubled by a disturbed motoric activity of alimentary tract in the form of periodic diarrheas, which frequently vanish spontaneously. Diarrhea used to manifest at night. Diabetic patients and, in particular, patients with type 2 diabetes frequently manifest the small intestine bacterial outgrowth (SIBO). However, data on the topic is equivocal: some studies documented lower incidence of the syndrome than that manifested in general population and the authors linked the fact with alimentary interventions in patients with diabetes. ${ }^{24}$ 


\section{Diseases of large intestine connected with diabetes}

The principal variables promoting the development of colorectal carcinoma involve a high fat and high carbohydrate diet, resulting in overweight and obesity, disturbances in carbohydrate metabolism and hyperinsulinemia. Several scientific reports accentuate also the role of diabetes as an independent risk factor of developing colorectal carcinoma. ${ }^{25}$ Moreover, a positive relationship was documented between the value of $\mathrm{HbA}_{1 \mathrm{c}}$ and the incidence of intestinal polyps. ${ }^{26}$ Metformin, as the drug of the first-line therapy of type 2 diabetes, induces the reduction in hyperinsulinemia and reduces insulin resistance. Nevertheless, apart from its anti-hyperglycemic effects, it exerts also other metabolically favorable sequels. In the mechanism of AMP kinase activation, metformin inhibits aberrant crypt foci (ACF), the marker of colorectal carcinoma (CRC). ${ }^{27}$ Studies of Choe et al. in the group using metformin documented a significantly lower frequency of colorectal polyps, their significantly lower size, lower number of hyperplastic and villiform polyps $(\mathrm{p}=0.01)$, and less advanced cases of CRC. ${ }^{27}$ However, it should be mentioned that metformin administered for many years reduces the level of vitamin $B_{12}$. A similar effect is exerted by proton pump inhibitors (PPIs), commonly used in gastritis and, therefore, the control of the level and possible supplementation of vitamin $B_{12}$ represent an indispensable element of coordinated treatment with the drugs. ${ }^{28}$

\section{Liver and diabetes: Does diabetic microangiopathy of the liver exist?}

The main hepatic pathologies diagnosed in diabetic patients include simple fatty degeneration of the liver, most frequently linked to type 2 diabetes, obesity and lipid disturbances and nonalcoholic fatty hepatitis, leading to fibrosis and cirrhosis of the liver. In patients with type 1 diabetes, the syndrome of Mauriac may develop, characterized by hepatomegaly. Diabetic fibrosis of the liver represents a form of hepatic microangiopathy in which sinusoidal fibrosis of the liver takes place with no traits of fatty degeneration. Deposits of collagen in liver sinusoids and remodeling of basement membrane with a normal ultrasonographic pattern are typical for the disease. ${ }^{29}$ According to Hudacko et al., this type of complication is linked to diabetic microangiopathy, developing in patients with DM1 and DM2, particularly in those with prolonged diabetic anamnesis and coexisting complications, most frequently of diabetic nephropathy type ${ }^{30}$ Frequently, also chronic elevation of alkaline phosphatase activity is detected. ${ }^{29}$

Propensity for various types of infections also characterizes diabetic patients, including viral hepatitis. It was confirmed that DM2 develops more frequently in persons infected with HCV than in persons free of such an infection or in those with coexisting HBV infection. ${ }^{31}$ This is liked to the presence of insulin resistance, higher secretion of tumor necrosis factor alpha (TNF- $\alpha$ ), as well as to the direct effect of viral proteins on the effect of insulin. ${ }^{32,33}$ Numerous studies also confirmed the relationship between $\mathrm{HCV}$ infection and type 1 diabetes, although the incidence of such an infection is much lower than in type 2 diabetes, and its manifestation is mainly linked to the induction of autoimmune process during therapy with interferon. ${ }^{34,35}$

\section{Tumors in alimentary tract versus type of diabetes}

For years it has been known that the risk of developing a tumor in the alimentary tract is markedly higher in diabetic patients than in normal individuals. The relationship is based (in the case of persons with type 2 diabetes) on the manifestation of overweight and obesity, hyperinsulinemia and insulin resistance. The phenomena lead to elevated concentrations of IGF-1 which, in turn, stimulate uncontrolled cell proliferation, while inhibiting the process of apoptosis. Similarly, in patients with type 1 diabetes, exogenous hyperinsulinemia may lead to pathological hypertrophy of cells and tissues. In studies by Mannucci et al., the teratogenic effect of high doses of exogenous insulin was confirmed. ${ }^{36}$ Patients with type 2 diabetes significantly more frequently develop tumors of liver, pancreas, esophagus and large intestine (in the female population, rectal tumors are seen particularly frequently). They used to involve adenomas, in contrast to myomas, the incidence of which is comparable to that in general population. In patients with type 1 diabetes also more frequently manifestations of hepatic and pancreatic carcinoma are observed and any tumors in their in situ forms. ${ }^{37}$ A significant problem for physicians is posed by the manifestation of pancreatic cancer in persons with freshly diagnosed diabetes. In such cases, diabetes presents frequently a principal sign of the concealed disease. Such a risk affects most frequently lean individuals of a mature age, with no pronounced insulin resistance, in whom clinical pattern is not typical for type 1 diabetes, while the signs/symptoms appeared suddenly and manifest a pronounced intensity. However, imaging studies in this group of patients are conducted exclusively in patients with a high risk of pancreatic tumor. A significant risk of developing a pancreatic tumor is present also in persons with class 3 diabetes. In persons with chronic pancreatitis, the presence of cysts of over $3 \mathrm{~cm}$ in diameter, with a dilation of pancreatic duct and with the presence of solid structures linked to cyst wall or in its lumen, provides an indication for oncological control. ${ }^{38}$ In the year of 2009, in studies published by European Association for the Study of Diabetes (EASD), the suspicion was advanced that the application of long-term acting glargine analogue may augment the incidence of breast cancer. However, such results have not been confirmed in the Outcome Reduction 
with an Initial Glargine Intervention (ORIGIN) study, published in 2014. ${ }^{39}$ Neoplastic process was found to be inhibited by the principal drug used in therapy of type 2 diabetes, metformin. The drug, due to its involvement in the signaling pathway of LKB1 kinase (a controller of AMPactivated protein kinase - AMPK), exerts a confirmed anti-proliferative effect and forms a recognized neoplastic suppressor. Metformin effect is also linked to the ability of AMPK to maintain low levels of cell energy while the phosphorylation of p27KIP and tuberous sclerosis complex 2 (TSC2) proteins leads to the inhibition of signaling network proliferation. ${ }^{40}$ In Zwolle Outpatient Diabetes project Integrating Available Care studies (ZODIAC16), the application of metformin from the beginning of the disease was found to be associated with a lower mortality of patients due to tumors and the effect seemed to be dependent on the dose. ${ }^{41}$

Summing up the above, the scope of the pathology related to alimentary system in diabetic patients depends on the duration of diabetes, the extent of its metabolic equilibration, on manifestation and intensity of accompanying diseases. Current therapy of diabetes is based on achieving normal metabolic control and on screening toward commonly known principal complications of diabetes, such as retinopathy, neuropathy and diabetic nephropathy. Complications of diabetes may affect many other systems and organs, including in particular alimentary tract. Recognition of the complications and implementation of appropriate treatment requires that the doctor will take a holistic view of the patient and will individualize respective recommendations and the therapy.

\section{References}

1. International Diabetes Federation. IDF Diabetes Atlas, 8th ed. Brussels, Belgium: International Diabetes Federation; 2017

2. Shenoy MP, Puranik RS, Vanaki SS, et al. A comparative study of oral candidal species carriage in patients with type 1 and type 2 diabetes mellitus. J Oral Maxillofac Pathol. 2014;18(1):60-65.

3. Javed F, Ahmed H, Mehmood A, et al. Association between glycemic status and oral Candida carriage in patients with prediabetes. Oral Surg Oral Med Oral Pathol Oral Radiol. 2014;117(1):53-58.

4. Nelson R, Shlossman M, Budding L, et al. Periodontal disease and NIDDM in Pima Indians. Diabetes Care. 1990;13:836-840.

5. Tsai C, Hayes C, Taylor G. Glycemic control of type 2 diabetes and severe periodontal disease in the US adult population. Community Dent Oral Epidemiol. 2002;30:182-192.

6. Al-Khabbaz AK. Type 2 diabetes mellitus and periodontal disease severity. Oral Health Prev Dent. 2014;12:77-82.

7. Wang X, Han X, Guo X, Luo X, Wang D. The effect of periodontal treatment on hemoglobin a1c levels of diabetic patients: A systematic review and meta-analysis. PloS One. 2014;9(9):108412.

8. Białecka M, Niedzwiecki P, Ziółkiewicz DZ, Wierusz-Wysocka B. Tonsillectomy due to chronic tonsillitis improves metabolic control in type 1 diabetic patients. Clin Diabetes. 2013;2(6):208-212.

9. Agrawal S, Patel P, Agrawal A, et al. Metformin use and the risk of esophageal cancer in Barrett esophagus. South Med J. 2014;107(12): 774-779.

10. Lin SW, Freedman ND, Hollenbeck AR, et al. Prospective study of selfreported diabetes and risk of upper gastrointestinal cancers. Cancer Epidemiol Biomarkers Prev. 2011;20:954-961.

11. Frasca F, Pandini G, Sciacca $L$, et al. The role of insulin receptors and IGF-1 receptors in cancer and other disease. Arch Physiol Biochem. 2008;114:23-37.
12. Dixon JL, Copeland LA, Zeber JE, et al. Association between diabetes and esophageal cancer, independent of obesity, in the United States veterans affairs population. Dis Esophagus. 2015. doi:10.1111/ dote. 12402

13. Mearin F, Malagelada JR. Gastroparesis and dyspepsia in patients with diabetes mellitus. Eur J Gastroenterol Hepatol. 1995;7(8):717-723.

14. Bharucha AE, Kudva Y, Basu A, et al. Relationship between glycemic control and gastric emptying in poorly controlled type 2 diabetes. Clin Gastroenterol Hepatol. 2015;13:466-476.

15. Gourcerol G, Tissier F, Melchior C, et al. Impaired fasting pyloric compliance in gastroparesis and the therapeutic response to pyloric dilatation. Aliment Pharmacol Ther. 2015;41:360-367.

16. Tack J, Carbone F, Rotondo A. Gastroparesis. Curr Opin Gastroenterol. 2015;31(6):499-505.

17. Boronikolos GC, Menge BA, Schenker N, et al. Upper gastrointestinal motility and symptoms in individuals with diabetes, prediabetes and normal glucose tolerance. Diabetologia. 2015;58:1175-1182.

18. Bahadoran Z, Mirmiran P, Zarif-Yeaganeh M, Zojaji H, Azizi F. Helicobacter pylori stool antigen levels and serological biomarkers of gastric inflammation are associated with cardio-metabolic risk factors in type 2 diabetic patients. Endocrinol Metab. 2015;30:280-287.

19. Grover M, Bernard CE, Pasricha PJ, et al. Clinical-histological associations in gastroparesis: Results from the Gastroparesis Clinical Research Consortium. Neurogastroenterol Motil. 2012;24:531-539.

20. Choi KM, Kashyap PC, Dutta N, et al. CD206-positive M2 macrophages that express heme oxygenase-1 protect against diabetic gastroparesis in mice. Gastroenterology. 2010;138:2399-2409.

21. Barshop K, Staller K, Semler J, Kuo B. Duodenal rather than antral motility contractile parameters correlate with symptom severity in gastroparesis patients. Neurogastroenterol Motil. 2015;27:339-346.

22. He L, Sun Y, Zhu Y, et al. Improved gastric emptying in diabetic rats by irbesartan via decreased serum leptin and ameliorated gastric microcirculation. Genet Mol Res. 2014;13(3):7163-7172.

23. Short AP, Donaghue KC, Ambler G, et al. Screening for celiac disease in type 1 diabetes: A systematic Review. Pediatrics. 2015;136(1): 170-176.

24. Adamska A, Nowak M, Piłaciński S, et al. The prevalence incidence of small intestinal bacterial overgrowth (SIBO) in patients with diabetes. Clin Diabetes. 2015;4:5175-5182.

25. Suh S, Kang M, Kim MY, et al. Korean type 2 diabetes patients have multiple adenomatous polyps compared to non-diabetic controls. J Korean Med Sci. 2011;26:1196-1200.

26. Huang $X$, Fan $Y$, Zhang $H$, et al. Association between serum $\mathrm{HbA}_{1 c}$ levels and adenomatous polyps in patients with the type 2 diabetes mellitus. Minerva Endocrinol. 2015;40(3):163-167.

27. Cho YH, Ko BM, Kim SH, et al. Does metformin affect the incidence of colonic polyps and adenomas in patients with type 2 diabetes mellitus? Intest Res. 2014;12(2):139-145.

28. Purchiaroni F, Galli G, Annibale B. Metformin plus proton pump inhibitors therapy: The cobalamin deficiency challenge. Eur Rev Med Pharmacol Sci. 2015;19:2501-2502.

29. King RJ, Harrison L, Gilbey SG, et al. Diabetic hepatosclerosis: Another diabetes microvascular complication? Diabet Med. 2016;33(2):5-7. doi:10.1111/dme.1288

30. Hudacko RM, Sciancalepore JP, Fyfe BS. Diabetic microangiopathy in the liver: An autopsy study of incidence and association with other diabetic complications. Am J Clin Pathol. 2009;132:494-499.

31. Naing CMJ, Ahmed SI, Maung M. Relationship between hepatitis $C$ virus infection and type 2 diabetes mellitus: Meta-analysis. World J Gastroenterol. 2012;18:1642-1651.

32. Lecube A, Hernández C, Genescà J, Simó R. Proinflammatory cytokines, insulin resistance, and insulin secretion in chronic hepatitis C patients: A case-control study. Diabetes Care. 2006;29:1096-1101.

33. del Campo JA, García-Valdecasas M, Rojas L, Rojas A, Romero-Gómez $M$. The hepatitis $C$ virus modulates insulin signaling pathway in vitro promoting insulin resistance. PLOS One. 2012;7:47904.

34. Fabris $\mathrm{P}$, Betterle C, Floreani A, et al. Development of type 1 diabetes mellitus during interferon alfa therapy for chronic HCV hepatitis. Lancet. 1992;340:548.

35. Popescu C, Popescu GA, Arama V. Type 1 diabetes mellitus with dual autoimmune mechanism related to pegylated interferon and ribavirin 
treatment for chronic HCV hepatitis. J Gastrointestin Liver Dis. 2013;22:101-104.

36. Mannucci E, Monami M, Balzi D, et al. Doses of insulin and its analogues and cancer occurrence in insulin-treated type 2 diabetic patients type 2 diabetic patients. Diabetes Care. 2010;33:1997-2003.

37. Harding JL, Shaw JE, Peeters A, Cartensen B, Magliano DJ. Cancer risk among people with type 1 and type 2 diabetes: Disentangling true associations, detection bias, and reverse causation. Diabetes Care. 2015;38:264-270.

38. Dąbrowski A. Management of asymptomatic neoplastic cysts in pancreas: Summary of recommendations. 2015 American Gastroenterological Association. Med Prakt. 2016;2:58-60.
39. Bordeleau L, Yakubovich N, Dagenais GR, et al. The association of basal insulin glargine and/or n-3 fatty acids with incident cancers in patients with dysglycemia. Diabetes Care. 2014:37:1360-1366.

40. Libby G, Donnelly LA, Donnan PT, et al. New users of metformin are at low risk of incident cancer: A cohort study among people with type 2 diabetes. Diabetes Care. 2009;32:1620-1625.

41. Landman GW, Kleefstra N, Hateren KJ, et al. Metformin associated with lower cancer mortality in type 2 diabetes: ZODIAC16. Diabetes Care. 2010;33:322-326. 